\title{
INVERTIBLE DISJOINTNESS PRESERVING OPERATORS
}

\author{
by C. B. HUIJSMANS and B. DE PAGTER
}

(Received 7th August 1992)

\begin{abstract}
It is shown that an invertible disjointness preserving operator from a uniformly complete vector lattice onto a normed vector lattice has a disjointness preserving inverse and is necessarily order bounded.
\end{abstract}

1991 Mathematics subject classification: 46A40, 46B42, 47B65.

\section{Introduction}

Throughout this paper $E$ and $F$ are Archimedean vector lattices and all mappings under consideration are supposed to be linear. The operator $T: E \rightarrow F$ is called disjointness preserving ( $d$-homomorphism) whenever $x_{1} \perp x_{2}$ (i.e., $\left|x_{1}\right| \wedge\left|x_{2}\right|=0$ ) in $E$ implies $T x_{1} \perp T x_{2}$ in $F$.

In the problem section of [6] Y. A. Abramovich raises the following question: if $E, F$ are vector lattices and $T: E \rightarrow F$ is invertible and disjointness preserving, is $T^{-1}: F \rightarrow E$ disjointness preserving as well? The plausibility of an affirmative answer to this question (at least in the case that $E$ and $F$ are Banach lattices) is justified by a recent result of $\mathrm{K}$. Jarosz [8] who shows that a one-to-one disjointness preserving operator (separating in his terminology) from $C(X)$ onto $C(Y)$ (with $X$ and $Y$ compact Hausdorff spaces) is necessarily continuous (=norm bounded) and has therefore a disjointness preserving inverse.

The main purpose of the present paper is to generalize this result to the effect that an invertible disjointness preserving operator from a Banach lattice $E$ onto a normed vector lattice $F$ has a disjointness preserving inverse and is necessarily norm bounded. Actually we shall consider a somewhat more general situation when $E$ is a uniformly complete vector lattice, $F$ is a normed vector lattice and $T: E \rightarrow F$ is bijective and disjointness preserving and we will prove that $T^{-1}: F \rightarrow E$ is disjointness preserving as well and moreover that $T$ is order bounded.

In [2] Y. A. Abramovich, A. I. Veksler and A. V. Koldunov introduce the so-called $d$ isomorphisms $\left(T: E \rightarrow F\right.$ is termed a $d$-isomorphism whenever $x_{1} \perp x_{2}$ in $E \Leftrightarrow T x_{1} \perp T x_{2}$ in $F$ ). Observe that a $d$-isomorphism is necessarily injective. Surjective $d$-isomorphisms are precisely the invertible disjointness preserving operators for which the inverse is also disjointness preserving. In Theorems 4, 5 and Added in Proof of the same paper [2] it is stated that a $d$-isomorphism $T$ from a Banach lattice onto a normed vector lattice is norm bounded. Our result shows that the assumption that $T^{-1}$ is disjointness preserving is superfluous. 
Finally, we prove that any invertible disjointness preserving operator from a discrete vector lattice onto an arbitrary vector lattice has a disjointness preserving inverse.

For the sake of simplicity we restrict our attention in this paper to real vector lattices. The extension to complex vector lattices is obtained via standard arguments. For unexplained terminology and unproved results we refer to the standard textbooks $[3,9$, $11,15,16]$.

\section{Preliminaries}

In this section we collect some properties of disjointness preserving operators which will play an important role in the sequel.

Once more, let $E, F$ be (Archimedean) vector lattices. It is easily verified and wellknown that $T: E \rightarrow F$ is disjointness preserving if and only if $|T x|=|T| x \|$ for all $x \in E$. This implies immediately that $T$ is already disjointness preserving whenever $x_{1} \wedge x_{2}=0$ in $E$ implies $T x_{1} \perp T x_{2}$ in $F$.

The following result, due to $M$. Meyer [12], deals with a description of order bounded disjointness preserving operators (called Lamperti operators by $W$. Arendt in [4]). For more elementary proofs, see S. J. Bernau [5] and B. de Pagter [14].

Theorem 1.1. Let $E, F$, be vector lattices and $T: E \rightarrow F$ order bounded and disjointness preserving.

(i) There exist lattice homomorphisms $T^{+}, T^{-}: E \rightarrow F$ such that $T=T^{+}-T^{-}$. Furthermore,

$$
T^{+} x=(T x)^{+}, T^{-} x=(T x)^{-}\left(x \in E^{+}\right)
$$

and hence $(T x)^{+} \wedge(T y)^{-}=0\left(x, y \in E^{+}\right)$.

(ii) The modulus $|T|$ exists, $|T|$ is a lattice homomorphism, $|T|=T^{+}+T^{-}$and

$$
|T x|=\|T|x|=|T| x\|=|T||x|(x \in E) .
$$

In fact, both (i) and (ii) characterize order bounded disjointness preserving operators. Notice that a disjointness preserving operator is therefore order bounded if and only if it is regular. In the next proposition we present another characterization of order bounded disjointness preserving operators. It is due to W. Arendt [4, Theorem 2.4] in the case that $E, F$ are Banach lattices.

Proposition 1.2. Let $E, F$, be vector lattices and $T: E \rightarrow F$. Then the following are equivalent.

(i) $T$ is order bounded and disjointness preserving.

(ii) $\left|x_{1}\right| \leqq\left|x_{2}\right|$ in $E$ implies $\left|T x_{1}\right| \leqq\left|T x_{2}\right|$ in $F$. 
Proof. (i) $\Rightarrow$ (ii). By Theorem $1.1,|T|$ exists. Since $|T| \geqq 0,\left|x_{1}\right| \leqq\left|x_{2}\right|$ implies $|T|\left|x_{1}\right|$ $\leqq|T|\left|x_{2}\right|$, i.e., $\left|T x_{1}\right| \leqq\left|T x_{2}\right|$.

(ii) $\Rightarrow$ (i). Obviously, (ii) implies the order boundedness of $T$. Moreover, if $\left|x_{1}\right|=\left|x_{2}\right|$ in $E$, then $\left|T x_{1}\right|=\left|T x_{2}\right|$ in $F$. Choosing $x_{1}=x(x \in E)$ and $x_{2}=|x|$ we get that $|T x|=|T| x||$ for all $x \in E$, so $T$ is disjointness preserving.

The next theorem has been proved by P. T. N. McPolin and A. W. Wickstead in [10, Theorem 2.1]. For previous versions of this result involving two sequences instead of one we refer to Y. A. Abramovich [1] and B. de Pagter [14].

Theorem 1.3. Let $E, F$ be vector lattices and $T: E \rightarrow F$ disjointness preserving. If, for every sequence $\left\{x_{n}\right\}_{n=1}^{\infty}$ in $E^{+}$which converges to 0 relatively uniformly, $\bigwedge_{n=1}^{\infty}\left|T x_{n}\right|=0$, then $T$ is order bounded.

It follows immediately that if $E$ and $F$ are normed vector lattices and $T: E \rightarrow F$ is norm bounded and disjointness preserving then $T$ is order bounded. Conversely, if $E$ is a Banach lattice, $F$ is a normed vector lattice and $T: E \rightarrow F$ is order bounded and disjointness preserving then $T$ is norm bounded, as any order bounded operator from a Banach lattice into a normed vector lattice is norm bounded. Hence, in the latter case order boundedness, norm boundedness and regularity are equivalent for disjointness preserving operators.

If $E, F$ are vector lattices and $T: E \rightarrow F$ is bijective, order bounded and disjointness preserving, then $|T|$ is a lattice isomorphism from $E$ onto $F$ implying that $T^{-1}: F \rightarrow E$ is also order bounded and disjointness preserving (cf. [7, Theorem 1]).

In [14] the second author introduces for every operator $T: E \rightarrow F$ ( $E, F$ vector lattices) the so-called ideal of order boundedness $A_{T}$ of $T$. By definition, $A_{T}$ is the union of all ideals $A$ in $E$ with the property that the restriction $T / A$ is order bounded. It is easily verified that $A_{T}$ is an ideal of $E$ and that $T / A_{T}$ is order bounded. Clearly, $A_{T}$ is then the largest ideal in $E$ on which $T$ is order bounded and it is not difficult to see that

$$
A_{T}=\{x \in E: T[0,|x|] \text { is order bounded }\} .
$$

The following result stems from the same paper [14, Theorem 8 and Corollary 9] and will play a key role in our considerations.

Theorem 1.4. If $E$ is a uniformly complete vector lattice. $F$ is a normed vector lattice and $T: E \rightarrow F$ is disjointness preserving, then $A_{T}$ is order dense in $E$ (i.e., for every $0<x \in E$ there exists $y \in A_{T}$ such that $\left.0<y \leqq x\right)$.

\section{The main results}

In this section we present the main result of this paper (Corollary 2.2 and Theorem 2.3). 
Theorem 2.1. If $E$ is a uniformly complete vector lattice, $F$ is a normed vector lattice and $T: E \rightarrow F$ is injective and disjointness preserving, then $T x_{1} \perp T x_{2}$ implies $x_{1} \perp x_{2}$ in $E$.

Proof. Since $\left|T x_{1}\right|=|T| x_{1}||$ and $\left|T x_{2}\right|=|T| x_{2}||$ we may assume without loss of generality that $x_{1} \geqq 0$ and $x_{2} \geqq 0$. Suppose contrary to what we claim that $x_{1} \wedge x_{2}>0$. The order denseness of $A_{T}$ (Theorem 1.4) yields that $0<w \leqq x_{1} \wedge x_{2}$ for some $0<w \in A_{T}$. By the Archimedean property of $E$ there exists $\lambda>1$ such that $y=\left(\lambda w-x_{1}-x_{2}\right)^{+}>0$ and certainly $y \in A_{T}$ as $y \leqq \lambda w$. The latter implies also that $y \leqq \lambda\left(x_{1} \wedge x_{2}\right)$. Put $u=\lambda w \wedge x_{1} \in A_{T}$ and $v=\lambda w \wedge x_{2} \in A_{T}$. Since $y \leqq \lambda w$, the inequalities

$$
y \wedge x_{1} \leqq u, y \wedge x_{2} \leqq v
$$

are obvious.

Observe that it follows from

$$
0 \leqq x_{1}-u=\left(x_{1}-\lambda w\right)^{+} \leqq\left(x_{1}+x_{2}-\lambda w\right)^{+}
$$

and $y \perp\left(x_{1}+x_{2}-\lambda w\right)^{+}$that

$$
y \wedge x_{1} \perp\left(x_{1}-u\right), y \wedge x_{2} \perp\left(x_{2}-v\right)
$$

(the second statement in (2) can be verified in a similar fashion). Next we show that

$$
\left|T\left(y \wedge x_{1}\right)\right| \leqq\left|T x_{1}\right|,\left|T\left(y \wedge x_{2}\right)\right| \leqq\left|T x_{2}\right|
$$

Indeed, since $T$ is order bounded and disjointness preserving on $A_{T}$, Proposition 1.2 and (1) imply $\left|T\left(y \wedge x_{1}\right)\right| \leqq|T u|$. By (2), $T\left(y \wedge x_{1}\right) \perp T\left(x_{1}-u\right)$. Hence

$$
\left|T\left(y \wedge x_{1}\right)\right| \leqq|T u| \leqq\left|T x_{1}\right|+\left|T\left(x_{1}-u\right)\right|
$$

gives

$$
\begin{aligned}
\left|T\left(y \wedge x_{1}\right)\right| & =\left(\left|T x_{1}\right|+\left|T\left(x_{1}-u\right)\right|\right) \wedge\left|T\left(y \wedge x_{1}\right)\right| \\
& \leqq\left|T x_{1}\right| \wedge\left|T\left(y \wedge x_{1}\right)\right| \leqq\left|T x_{1}\right|
\end{aligned}
$$

The second inequality in (3) can be treated analogously.

Since $\left|T x_{1}\right| \wedge\left|T x_{2}\right|=0$, it is an immediate consequence of (3) that

$$
T\left(y \wedge x_{1}\right) \perp T\left(y \wedge x_{2}\right)
$$

Furthermore, since $\lambda>1$ and $y \leqq \lambda\left(x_{1} \wedge x_{2}\right)$ we clearly have

$$
y \wedge x_{1} \leqq \lambda\left(y \wedge x_{2}\right)
$$


Since $T$ is order bounded on $A_{T}$, (5) gives

$$
\left|T\left(y \wedge x_{1}\right)\right| \leqq \lambda\left|T\left(y \wedge x_{2}\right)\right| .
$$

Combining this with (4) we get $T\left(y \wedge x_{1}\right)=0$. But $T$ is one-one, so $y \wedge x_{1}=0$. On the other hand, $0<y \leqq \lambda w \leqq \lambda x_{1}$, a contradiction. It follows that $x_{1} \wedge x_{2}=0$ and the proof is complete.

Corollary 2.2. If $E$ is a uniformly complete vector lattice, $F$ is a normed vector lattice and $T: E \rightarrow F$ is bijective and disjointness preserving, then $T^{-1}: F \rightarrow E$ is disjointness preserving as well.

Theorem 2.3. If $E$ is a uniformly complete vector lattice, $F$ is a normed vector lattice and $T: E \rightarrow F$ is an invertible disjointness preserving operator, then $T$ is order bounded.

Proof. We have to show that $\left|x_{1}\right| \leqq\left|x_{2}\right|$ in $E$ implies $\left|T x_{1}\right| \leqq\left|T x_{2}\right|$ in $F$. Using that $\left|T x_{1}\right|=|T| x_{1}||,\left|T x_{2}\right|=|T| x_{2}||$ we may assume $0 \leqq x_{1} \leqq x_{2}$.

First we claim that for each $0 \leqq y \in A_{T}$

$$
\left(\left|T x_{1}\right|-\left|T x_{2}\right|\right)^{+} \perp T\left(\left(y-x_{2}\right)^{+}\right) .
$$

Let $u=y \wedge x_{1}$ and $v=y \wedge x_{2}$. Then $0 \leqq u \leqq v \in A_{T}$ and hence $|T u| \leqq|T v|$. Since $x_{1}-u=$ $\left(x_{1}-y\right)^{+} \leqq\left(x_{2}-y\right)^{+}$and $x_{2}-v=\left(x_{2}-y\right)^{+}$, we have $x_{1}-u \perp\left(y-x_{2}\right)^{+}$as well as $x_{2}-v \perp$ $\left(y-x_{2}\right)^{+}$. Consequently,

$$
T x_{1}-T u \perp T\left(\left(y-x_{2}\right)^{+}\right), T x_{2}-T v \perp T\left(\left(y-x_{2}\right)^{+}\right) .
$$

Using one of the Birkhoff inequalities (that $\left|a^{+}-b^{+}\right| \leqq|a-b|$ for all $a, b \in F$ ) and the triangle inequality we get

$$
\begin{aligned}
& \left(\left|T x_{1}\right|-\left|T x_{2}\right|\right)^{+} \\
& =\left|\left(\left|T x_{1}\right|-\left|T x_{2}\right|\right)^{+}-(|T u|-|T v|)^{+}\right| \\
& \leqq\left|T x_{1}\right|-\left|T x_{2}\right|-(|T u|-|T v|) \mid \\
& \leqq\left|T x_{1}-T u\right|+\left|T x_{2}-T v\right| .
\end{aligned}
$$

This inequality together with (8) yields (7).

Next we claim that the set

$$
B=\left\{T\left(\left(y-x_{2}\right)^{+}\right): 0 \leqq y \in A_{T}\right\}
$$


is order dense in $F$. To this end, pick $0 \leqq q \in F$ such that $q \perp T\left(\left(y-x_{2}\right)^{+}\right)$for all $0 \leqq y \in A_{T}$. Since $T$ is surjective there exists $0 \leqq p \in E$ that satisfies $q=|T p|$, so $T p \perp$ $T\left(\left(y-x_{2}\right)^{+}\right)$for all $0 \leqq y \in A_{T}$. By Theorem 2.1, $p \wedge\left(y-x_{2}\right)^{+}=0$ for all $0 \leqq y \in A_{T}$. But $A_{r}$ is order dense in $E$ by Theorem 1.4, so $p \wedge\left(z-x_{2}\right)^{+}=0$ for all $0 \leqq z \in E$. Choosing $z=p+x_{2}$ we find that $p=0$ and hence $q=0$, showing that $B$ is order dense in $F$. By (7), $\left(\left|T x_{1}\right|-\left|T x_{2}\right|\right)^{+}$is disjoint from $B$ and so $\left(\left|T x_{1}\right|-\left|T x_{2}\right|\right)^{+}=0$, i.e., $\left|T x_{1}\right| \leqq\left|T x_{2}\right|$ and we are done.

Corollary 2.4. If $E$ is a Banach lattice, $F$ is a normed vector lattice and $T: E \rightarrow F$ is bijective and disjointness preserving, then $T$ is norm bounded (and $T^{-1}$ is disjointness preserving).

The following example (obtained jointly with A. W. Wickstead) shows that the uniform completeness of $E$ in Theorem 2.3 is not redundant. It is modification of an example due to $M$. Meyer [13].

Example 2.5. Let $E=F=P L[0,1)$, the vector lattice of all piecewise linear functions on $[0,1)$, i.e., $f \in P L[0,1)$ if and only if there exists a partition

$$
0=x_{0}<x_{1}<\cdots<x_{n-1}<x_{n}=1
$$

( $n$ depending on $f$ ) such that $f$ is linear on each interval $\left[x_{i-1}, x_{i}\right)(i=1, \ldots, n)$.

Observe that $P L[0,1)$ is a normed vector lattice (with respect to the supremum norm) that has the principal projection property, but is not uniformly complete. Define $D$ : $P L[0,1) \rightarrow P L[0,1)$ by

$$
(D f)(x)=f_{r}^{\prime}(x)(0 \leqq x<1, f \in P L[0,1)),
$$

where $f_{r}^{\prime}$ stands for the right derivative of $f$. Observe that $D$ is band preserving (so certainly disjointness preserving), not order bounded and that $D^{2}=0$, Let $T=I+D$. Then $T$ is bijective, $T^{-1}=I-D$ and hence $T$ is an invertible disjointness preserving operator which is not order bounded. Notice that both $T$ and $T^{-1}$ are even band preserving.

In our final result we shall describe one more situation in which any invertible disjointness preserving operator has a disjointness preserving inverse.

Theorem 2.6. If $E$ and $F$ are vector lattices, $E$ is discrete and $T: E \rightarrow F$ is invertible and disjointness preserving, then $T^{-1}: F \rightarrow E$ is also disjointness preserving.

Proof. By hypothesis, $E$ contains a maximal disjoint system of atoms $\left\{e_{\sigma}\right\}_{\sigma \in S}$. The band $\left\{e_{\sigma}\right\}^{d d}$ generated by $e_{\sigma}$ is a projection band and satisfies

$$
\left\{e_{a}\right\}^{d d}=\left\{\alpha e_{\sigma}: \alpha \in \mathbf{R}\right\} .
$$

Denote the band projection of $E$ onto $\left\{e_{\sigma}\right\}^{d d}$ by $P_{\sigma}(\sigma \in S)$. We have to show that 
$x_{1}, x_{2} \in E^{+}, T x_{1} \perp T x_{2}$ implies $x_{1} \wedge x_{2}=0$. Since $P_{\sigma} x_{1} \perp x_{1}-P_{\sigma} x_{1}$ and $T$ is disjointness preserving we have $T P_{\sigma} x_{1} \perp T\left(x_{1}-P_{\sigma} x_{1}\right)$ (actually we use here that $T$ maps components onto components! $)$. Hence. $T x_{1}=T P_{\sigma} x_{1}+\left(T x_{1}-T P_{\sigma} x_{1}\right)$ implies $\left|T x_{1}\right|=\left|T P_{\sigma} x_{1}\right|+$ $\left|T x_{1}-T P_{\sigma} x_{1}\right|$ and thus $\left|T P_{\sigma} x_{1}\right| \leqq\left|T x_{1}\right|$. Similarly $\left|T P_{\sigma} x_{2}\right| \leqq\left|T x_{2}\right|$. Therefore $\left|T P_{\sigma} x_{1}\right| \wedge$ $\left|T P_{\sigma} x_{2}\right|=0$, as $T x_{1} \perp T x_{2}$. On the other hand, $P_{\sigma} x_{1}=\alpha e_{\sigma}, P_{\sigma} x_{2}=\beta e_{\sigma}$ for appropriate $\alpha, \beta \in \mathbf{R}$. Consequently, $T P_{\sigma} x_{1}=\alpha T e_{\sigma}$ and $T P_{\sigma} x_{2}=\beta T e_{\sigma}$. Since $\alpha T e_{\sigma} \perp \beta T e_{\sigma}$ and $T e_{\sigma} \neq 0$ (because $T$ is one-one) we find $\alpha=0$ or $\beta=0$ which results in $P_{\sigma} x_{1}=0$ or $P_{\sigma} x_{2}=0$. It follows that $P_{\sigma}\left(x_{1} \wedge x_{2}\right)=P_{\sigma} x_{1} \wedge P_{\sigma} x_{2}=0$ for all $\sigma \in S$, i.e., $x_{1} \wedge x_{2} \wedge e_{\sigma}=0$ for all $\sigma$. But the band generated by $\left\{e_{\sigma}\right\}_{\sigma \in S}$ is the whole of $E$, so $x_{1} \wedge x_{2}=0$ which is the desired result.

The corresponding question for band preserving operators was dealt with by $\mathrm{A}$. W. Wickstead and the first author in [7], where they showed that the inverse of a bijective band preserving operator on a uniformly complete vector lattice is band preserving as well.

We are grateful to the referee for some valuable remarks.

\section{REFERENCES}

1. Y. A. Abramovich, Multiplicative representation of disjointness preserving operators, Indag. Math. 45 (1983), 265-279.

2. Y. A. Abramovich, A. I. Veksler and A. V. Koldunov, On operators preserving disjointness, Soviet Math. Dokl. 20 (1979), 1089-1093.

3. C. D. Aliprantis and O. Burkinshaw, Positive Operators (Academic Press, Orlando, 1985).

4. W. ARENDT, Spectral properties of Lamperti operators, Indiana Univ. Math. J. 32 (1983), 199-215.

5. S. J. Bernau, Orthomorphisms of Archimedean vector lattices, Math. Proc. Cambridge Philos. Soc. 89 (1981), 119-128.

6. C. B. Huismans and W. A. J. Luxemburg (eds.), Positive Operators and Semigroups on Banach Lattices (Kluwer, Dordrecht, 1992); also appeared in Acta Appl. Math. 27 (1992), 143-152.

7. C. B. Huismans and A. W. Wickstead, The inverse of band preserving and disjointness preserving operators, Indag. Math. 3 (N.S.) (1992), 179-183.

8. K. J J (1990), 139-144.

9. W. A. J. Luxemburg and A. C. ZaAnen, Riesz Spaces I (North-Holland, Amsterdam, 1971).

10. P. T. N. McPolin and A. W. WicksteAD, The order boundedness of band preserving operators on uniformly complete vector lattices. Math. Proc. Cambridge Philos. Soc. 97 (1985), 481-487.

11. P. Meyer-Nieberg, Banach Lattices (Springer, Universitext, Berlin, 1991).

12. M. Meyer, Le stabilisateur d'un espace vectoriel réticulé, C. R. Acad. Sci. Paris 283 (1976), 249-250.

13. M. MeYer, Quelques propriétés des homomorphisms d'espaces vectoriels réticulés, Equipe d'Analyse Paris VI, preprint 131, 1979. 
14. B. de Pagter, A note on disjointness preserving operators, Proc. Amer. Math. Soc. 90 (1984), 543-549.

15. H. H. Schaefer, Banach lattices and Positive Operators (Springer, Ergebnisse 215 (Berlin, 1974).

16. A. C. ZaAnen, Riesz Spaces II (North-Holland, Amsterdam, 1983).

Department of Mathematics

UNIVERSITY OF LEIDEN

P.O. Box 9512

2300 RA LEIDEN

THE NeTHERLANDS
Faculty of Technical Mathematics

and COMPuter SCIENCE

DelfT University of TeChNology

P.O. Box 356

2500 AJ Delft

THE NeTHERLANDS 